\title{
Clinical profile of Rickettsial fever in children in a government tertiary care institute in north Karnataka
}

\author{
Vishwanath Pattar ${ }^{1}$, Venkatesh $\mathrm{G}^{2 *}$, Shantharaja G $\mathrm{C}^{3}$, Srinivasa Arer ${ }^{4}$, Jayaraj Pati ${ }^{5}$, \\ Shivanagouda $\mathrm{J}^{6}$
}

${ }^{1,3} \mathrm{DCH}$ (CPS Mumbai)Post Graduate, ${ }^{2}$ Assistant Professor, ${ }^{4}$ Professor \& HOD, ${ }^{5,6}$ Associate Professor, Department of Paediatrics, Gadag Institute Medical Sciences Gadag, INDIA.

Email: shivanagoudajoladarasi@gmail.com

$\underline{\text { Abstract }}$

Background: Rickettsial diseases are a group of Febrile illnesses caused by obligate intracellular gram negative coccobacilli and transmitted to man by arthropod vectors., often under diagnosed due to poor awareness. Rickettsial Diseases are dangerous among children, due to atypical presentations and complications, as increasing trends in India since last many years. Objective: To assess the clinical Profile of Rickettsial fever among Children. Methodology: A prospective Cohort study was conducted in the Dept of Pediatrics, Gadag Institute of Medical Sciences Gadag Karnataka State, among the Children Attended OPD and Admitted in our Hospital, from the month of September 2019 to February 2020, a total of 131 cases reported during the study period have been included. Results: All cases Having H/O High grade Fever and Weil Felix anti body test, positive [131- 100\%] have been included. Among them $49\{39 \%\}$ are positive for Spotted fever(OX2) [ caused by Rickettsia ricketsii, conori], $22\{17 \%\}$ for Scrub typhus(OX-K) [ caused by Orienta Tsutsugamushi], 22 $\{17 \%\}$ for Endemic Typhus/Epidemic typhus (OX-19) [caused by Rickettsia Typhi, prowazeki], $27\{21 \%\}$ for both scrub Typhus and Endemic Typhus, 7(5\%) for both Scrub typhus and spotted fever, $1(0.78 \%)$ for both Endemic typhus and Spotted fever, 3(2.2\%) for all 3 antigens. All the cases have been treated with Doxycycline $2.8-3 \mathrm{mg} / \mathrm{kg} / \mathrm{day}$ BID doses for 7 to 10 days. Among them about, 33\% patients responded within 48 hours of treatment, $57 \%$ within 72 hours $10-12 \%$, recovered well within 5-6days of the treatment. Conclusion: Rickettsial fever does exist in our area and incidence is rising after the ending of rainy season peak during the month of September to January. Rickettsial fever should be kept in mind for workup of exanthematous fever.

Keywords: Rickettsial fever, Exanthematous fever, Weil Felix anti body test, Febrile illnesses.

\section{*Address for Correspondence:}

Dr Venkatesh G, Assistant Professor, Department of Paediatrics, Gadag Institute Medical Sciences, Gadag, INDIA.

Email: shivanagoudajoladarasi@gmail.com

Received Date: 02/04/2020 Revised Date: 10/05/2020 Accepted Date: 16/06/2020

DOI: https://doi.org/10.26611/10141512

This work is licensed under a Creative Commons Attribution-NonCommercial 4.0 International License. (cc)) EY-NC

\begin{tabular}{|c|c|}
\hline \multicolumn{2}{|c|}{ Access this article online } \\
\hline Quick Response Code: & Website: \\
& www.medpulse.in \\
\cline { 2 - 2 } & \\
\hline
\end{tabular}

\section{INTRODUCTION}

Rickettsial diseases are a group of Febrile illnesses caused by obligate intracellular gram-negative coccobacilli and transmitted to man by arthropod vectors., often under diagnosed due to poor awareness. . $, 2,3^{2}$
In recent years outbreaks have been reported in the sub Himalayan Belts, Maharashtra, Rajasthan, Punjab, Tamil Nadu, Kerala, Karnataka ${ }^{4,5,6,7,8,9}$ and Rickettsial diseases are important and often under recognized cause of febrile illnesses in children with wide variety of atypical multisystem clinical manifestations. ${ }^{10,11}$

The family of Rickettsia is named after HOWARD TAYLOR Ricketts who discovered Spotted fever and died during his studies in 1909. ${ }^{12,13}$ Rickettsial are group of motile, gram negative, non-spore forming, highly pleomorphic bacteria present as coccobacilli, or thread like intracellular obligate parasites., divided epidemiologically into 4 groups. ${ }^{1,2}$

$\begin{array}{lrrr}\text { 1. Spotted fever } & \text { group(Rocky } & \text { mountain } & \text { spotted } \\ \text { fever[Rickettsia } & \text { ricketsii], } & \text { Indian } & \text { tick }\end{array}$ typhus,[Rickettsia Conori] 
2. Typhus fever group (Epidemic louse born typhus,[Rickettsia prowazeki] Endemic/murine Typhus,) [Rickettsia typhi]

3. Scrub Typhus[[ Orientia Tsutsugamushi]

4. Miscellaneous [TIBOLA -Tick born Lymphadenopathy, caused by Ehrlichiosis @ Anaplasmosis]

Spread by Arthropod vectors, lice, fleas, ticks, mites are the most frequent presenting symptoms include, high grade fever, headache, rashes, myalgia, anorexia, nausea, vomiting, diarrhea, abdominal pain., and complicated cases may have multi organ involvement -encephalopathy, pulmonary edema, acute renal failure, vascular collapse, myocarditis, hepatic failure. ${ }^{1,2,13,14}$ Rickettsial Diseases are dangerous among children, due to atypical presentations and complications, as increasing trends in India since last many years. ${ }^{2,13,14} \mathrm{We}$ in the north Karnataka zone met across with more endemic cases of Rickettsial diseases, coming from rural and urban areas of Gadag district, in the month of September and October, as we don't have much study data on Rickettsial fever in our District area, thus we conducted a study to evaluate the disease presentation, profile and prevalence of organism causing disease, among children attended OPD and admitted in our hospital. This study aims clinical suspicion, awareness about transmission, and laboratory evaluation, treatment and

\section{METHEDOLOGY}

A prospective Cohort study was conducted in the Dept of Pediatrics, Gadag Institute of Medical Sciences Gadag Karnataka State, among the Children Attended OPD and Admitted in our Hospital, from the month of September 2019 to February 2020, a total of 131 cases reported during the study period have been included. Base line Data of age, sex, local residing area, exposure to animals, Socio economic status, date of admission, days onset of first symptom fever/ rash , distribution of rash, associated other systemic co morbidities, were recorded, and done $\mathrm{CBC}$ of all patients, weil Felix test to identify the type of species,

\section{Procedure of weil Felix test}

The Weil-Felix test is based on the detection of antibodies to various cross-reacting Proteus antigens with Rickettsia (Proteus vulgaris OX2 with spotted fever Rickettsiae, P. vulgaris OX19 with typhus group Rickettsiae and Proteus mirabilis OXK with O.Tsutsugamushi). The Weil-Felix test was performed on all the serum samples collected at the time of presentation at the hospital (acute sample) $)^{13}$ Done by Slide test method using with PROGEN- Proteus antigen suspensions for weil Felix test. [Manufactured by TULIP DIAGNOSTICS (P) LTD, Verna Goa.] packed with 3 separate containers of $5 \mathrm{ml}$ of Reagents of OX-K [scrub typhus], OK-19 [epidemic typhus] and OX-2 [scrub typhus].
A clean white background reaction circle glass slide is taken, place one drop of Positive control, physiological saline, and patient serum to be tested in each circle of the slide, then put 1 drop of appropriate PROGEN antigen to each circle, mix with separate mixture sticks wait for 1 minute, and see the level of agglutinins in serum circle, positive agglutinins indicates presence of Rickettsial antibodies in the patient serum, Agglutination titer of 1:80 or more was considered significant for Rickettsial infection. And also we done LFT, RFT, Serum electrolytes, peripheral smears, dengue card test, for necessary patients, and treated with Doxycycline 2.5-3 $\mathrm{mg} / \mathrm{kg}$ BD doses for 7-10 days.

\section{INCLUSION CRITERIA}

Clinically suspected cases with $\mathrm{h} / \mathrm{o}$ fever, macula popular, Erythematous rash, in children with age group of 6months to 18years with Positive weil Felix test for - OX-19, OX2 , and $\mathrm{OX}$ -

\section{EXCLUSION CRITERIA}

Fever and Rashes with Dengue Positive, Leptospirosis, Brucella infections

Statistical Analysis: Data was entered in Microsoft Excel and analyzed using SPSS version 24.0th. Mean and Standard deviation were calculated for quantitative variables, and proportion calculated for categorical variables.

Ethical committee approval taken from Institution.

\section{RESULTS}

In our study duration of 6 months, from Sep2019 to Feb 2020, included 131 [51 OPD (38.9\%) + 80 IPD (61.01\%) Cases] children of age group 6 months to 18 years, in which 71 are Male (54.19\%) 60 are Female (45.81\%). Highest Burden of the disease occurred during Sep and Oct months [71 cases $54.19 \%$ ] soon after rainy season, among them 48 Cases [36.9\%] have h/o animal contact [Dog, goat, cow, Buffalo, and insect bites; Rat flea], 12cases $[9.1 \%]$ have $\mathrm{h} / \mathrm{o}$ Lice infestation.

All cases Having H/O High grade Fever and Weil Felix anti body test, positive [131- 100\%] have been included. Among them $49\{39 \%\}$ are positive for Spotted fever(OX2) [ caused by Rickettsia ricketsii, conori], $22\{17 \%\}$ for Scrub typhus(OX-K) [ caused by OrientaTsutsugamushi], $22\{17 \%\}$ for Endemic Typhus/Epidemic typhus (OX-19) [ caused by Rickettsia Typhi, prowazeki], $27\{21 \%\}$ for both scrub Typhus and Endemic Typhus, 7 (5\%) for both Scrub typhus and spotted fever, $1(0.78 \%)$ for both Endemic typhus and Spotted fever, 3(2.2\%) for all 3 antigens.

84 cases [64.1\%] have Purpuric Rashes, Hepatomegaly 23 $[17.5 \%]$, GI Upset 78 [59.5\%], Seizures and Encephalopathy 5 [3.8\%], Malaise, Body ache 42 [32\%], Cough and Pneumonia 46 [35\%] are present. 
Distribution of Prevalence of Type of Rickettsial species is shown in the Pie Chart below.

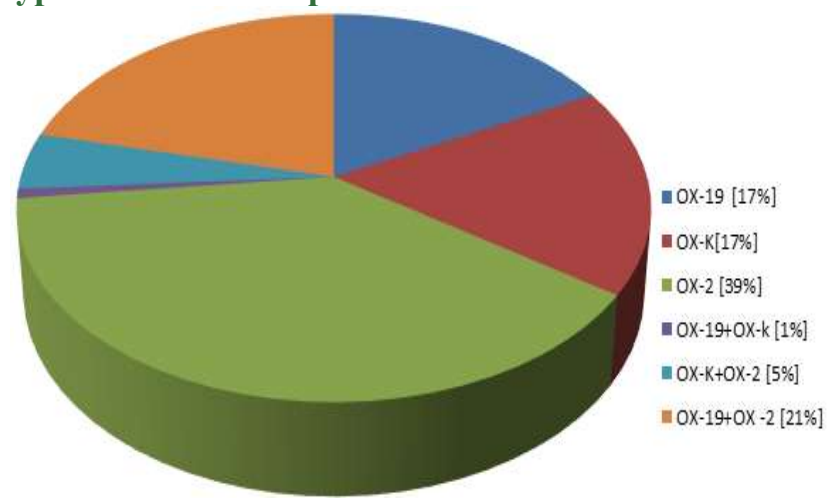

Figure 1: Weil felix test interpretation

The following Table no 1 shows multi systemic clinical manifestations of the reported cases and Table no 2 shows Laboratory investigations. Table no 3 other lab investigations

Table 1: Major Presenting symptoms

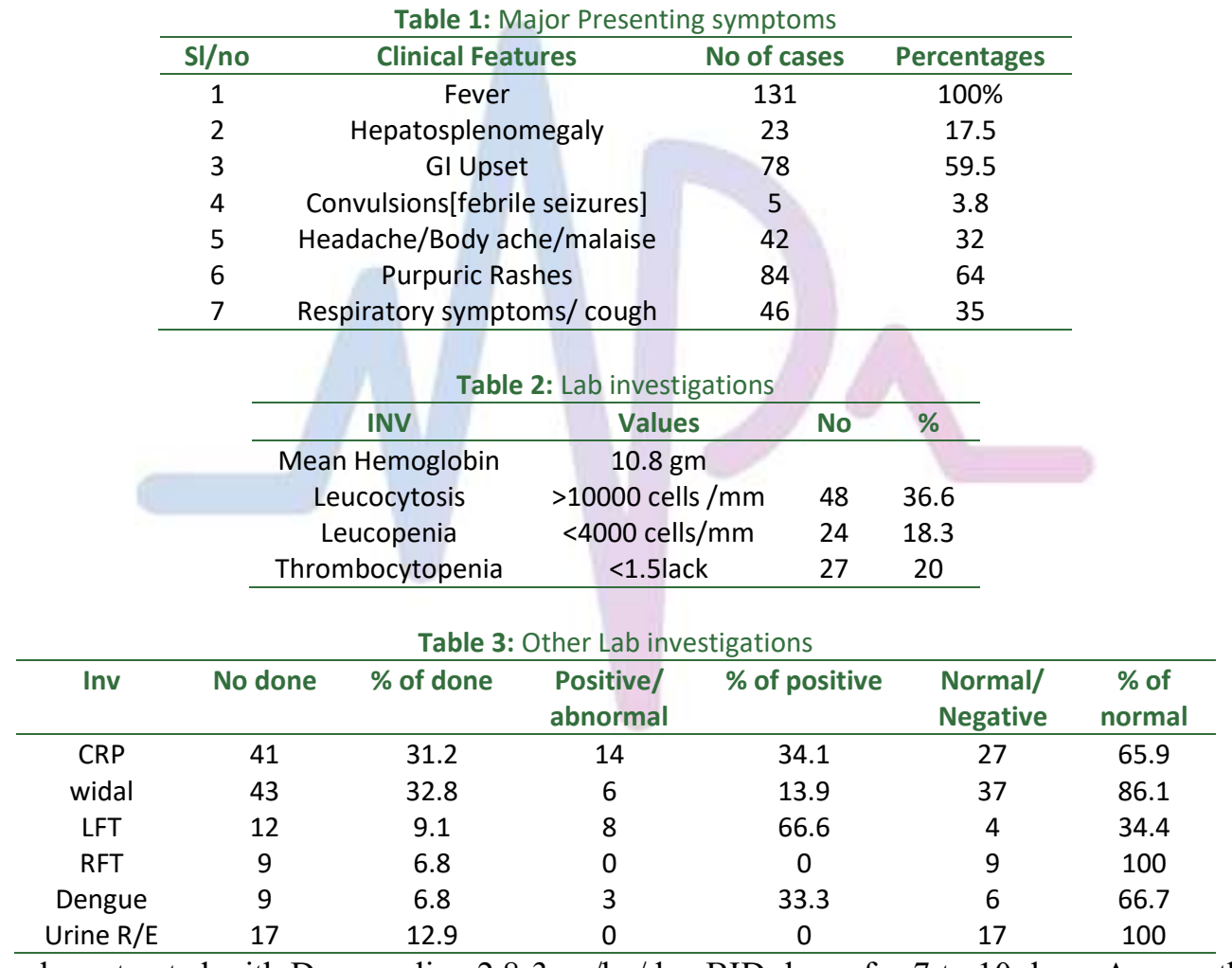

All the cases have been treated with Doxycycline 2.8-3mg/kg/day BID doses for 7 to 10 days. Among them about, 33\% patients responded within 48 hours of treatment, 57\% within 72 hours $10-12 \%$ recovered well within 5-6days of the treatment.

\section{DISCUSSION}

Rickettsial Diseases are one of the important cause for Febrile illnesses in children with wide variety of clinical manifestations, often under diagnosed due to poor awareness. In our study we have shown the disease profile and prevalence of type of Rickettsial species [strain] in our area. In our study age group ranged from 6months to 18 years, with mean age of 7.5 year, with no statistically significant sex difference. Which is similar to Kalal B $\mathrm{S}^{14}$, 6 months to 12 years taken mean age 7.4 years and Sunil S. Vaidya $^{13},<18$ years with mean age 7 years. All the patients presented with fever[100\%], Maculopapular purpuric rashes seen in $64 \%$, which is $82 \%$ in Sunil S Vaidya ${ }^{13}, 36 \%$ in Kalal B S ${ }^{14}$, GI symptoms, $59.5 \%$, which is $25 \%$ in Sunil S Vaidya ${ }^{13}, 44 \%$ in Kalal B S ${ }^{14}$, Hepatomegaly $17.5 \%$, which is $65 \%$ in Sunil S Vaidya ${ }^{13}, 71 \%$ in Kalal B S ${ }^{14}$, 
CNS symptoms $3.8 \%$, which is $34 \%$ in Sunil S Vaidya ${ }^{13}$, $15 \%$ in Kalal B S ${ }^{14}$, Resp symptoms $35 \%$, which is $5 \%$ in Sunil S Vaidya ${ }^{13}$, Headache/malaise $32 \%$, which is $10 \%$ in Sunil S Vaidya. ${ }^{13}$ In our Study found, Leukocytosis $36.6 \%$, which is $66 \%$ in Sunil S Vaidya ${ }^{13}$, Thrombocytopenia $20 \%$, which is $56 \%$ in Sunil S Vaidya. ${ }^{13}$ In our Study according to Weil felix test among $\{39 \%\}$ are positive for Spotted fever[ caused by Rickettsia ricketsii, conori], is prevalent in our area, $22\{17 \%\}$ for Scrub typhus [ caused by Orienta Tsutsugamushi], $\{17 \%\}$ for Endemic Typhus/Epidemic typhus [caused by Rickettsia Typhi, prowazeki], $27\{21 \%\}$ for both scrub Typhus and Endemic Typhus, whereas Tick born spotted is or epidemic typhus is prevalent in in Sunil S Vaidya ${ }^{13}$, and Scrub typhus $85.5 \%, 37.1 \%$ Spotted fever in in Kalal B S ${ }^{14}$, Scrub typhus $80 \%$ in Thomos R, et al. ${ }^{15}$ As the weil felix test may give false positive cross reaction with proteus species, leptospira, Borelia infections, a definitive test like ELISA or PCR should be done to confirm the specific Rickettsial strains. In some study centers used Chloramphenicol or Doxycycline for treatment; we used only Doxycycline and are responded well in all the cases.

\section{CONCLUSION}

Rickettsial fever does exist in our area and incidence is rising after the ending of rainy season peak during the month of September to January. Rickettsial fever should be kept in mind for workup of exanthematous fever. Early diagnosis and treatment with Doxycycline can reduce the hospital stay and cost. Use of Empherical treatment may be considered to reduce the morbidity and mortality observed with the disease.

Limitation of the study

As the Weil Felix test not Diagnostic standard, it should be interpreted in good clinical context, still easily available to all and remain good screening test. Investigation like PCR should done to detect and Strengthen the diagnosis of Rickettsial organism.

\section{REFERENCES}

1. Ghai Essential Pediatrics, $9^{\text {th }}$ Edition chapter 11, PN- 251.

2. PG Textbook of Pediatrics, Piyush Gupta, $2^{\text {nd }}$ edition volume 2, chapter 34.1, PN-1558

3. Mukhesh Agarwal Text book of Pediatrics.

4. Rathi N,Rathi A. Rickettsial infections: Indian Prospective . Indian Pediatrics 2010; 47:157-64

5. Rapsang AG, Bhattacharyya P. Scrub typhus. Indian J Anaesth 2013; 57:127-34

6. Rahi M, Gupte MD, Bhargava A, Varghese GM, Arora R. DHR-ICMR Guidelines for diagnosis and management of Rickettsial diseases in India. Indian J Med Res 2015; 141:417-22.

7. Ramyasree A, Kalawat U, Rani ND, Chaudhury A. Seroprevalence of scrub typhus at a tertiary care hospital in Andhra Pradesh. Indian J Med Microbiol 2015; 33:6872.

8. Mahajan SK, Rolain JM, Kashyap R, Bakshi D, Sharma V, Prasher BS, et al... Scrub typhus in Himalayas. Emerg Infect Dis 2006; 12:1590-2.

9. Somashekar HR, Moses PD, Pavithran S, Mathew LG, Agarwal I, Rolain JM, et al... Magnitude and features of scrub typhus and spotted fever in children in India. J Trop Pediatr 2006; 52:2289

10. Batra HV. Spotted fevers and typhus fever in Tamil Nadu. Indian J Med Res 2007; 126:101-3.

11. Kulkarni A. Childhood rickettsiosis. Indian J Pediatr 2011; 78:81-7.

12. Raoult D, Parola P, editors. Rickettsial Diseases. New York : informa Healthcare USA 2007.[PubMed]

13. Sunil S Vaidya, Atul kulakarni, clinical study and laboratory profile of Rickettsial fever in children, a study from rural Maharashtra . Int J Pediatr Res.2016;3(8): 559562.

14. Kalal B S, Puranik P, Nagaraj S, Rego S, Shet A. Scrub typhus and spotted fever among hospitalized children in South India: Clinical profile and serological epidemiology. Indian J Med Microbiol [serial online] $2016 ; 34: 293-298$

15. Paris DH, Shelite TR, Day NP, Walker DH. Unresolved problems related to scrub typhus: a seriously neglected life-threatening disease. Am $J$ Trop Med Hyg. 2013;89(2):301-307. doi:10.4269/ajtmh.13-0064.

\section{Source of Support: None Declared
Conflict of Interest: None Declared}

Policy for Articles with Open Access:

Authors who publish with MedPulse International Journal of Pediatrics (Print ISSN: 2579-0897) (Online ISSN: 2636-4662) agree to the following terms: Authors retain copyright and grant the journal right of first publication with the work simultaneously licensed under a Creative Commons Attribution License that allows others to share the work with an acknowledgement of the work's authorship and initial publication in this journal.

Authors are permitted and encouraged to post links to their work online (e.g., in institutional repositories or on their website) prior to and during the submission process, as it can lead to productive exchanges, as well as earlier and greater citation of published work. 